\title{
On Robustness of a Class of Homogeneous Continuous Finite Time Controllers
}

\author{
Harshal B. Oza, Yury V. Orlov and Sarah K. Spurgeon
}

\begin{abstract}
This paper gives a Lyapunov based proof of robustness of a class of finite time controllers applied to the double integrator system. The literature of continuous finite time stabilisation contains the proof of finite time stability when continuous disturbances with a Lipschitz upper bound appear in the system dynamics. It is also known that continuous finite time controllers render the trajectories ultimately bounded for persisting disturbances. However, proving robustness of continuous finite time controllers to continuous disturbances with a non-Lipschitz upper bound is challenging. The main contribution of the paper is that it identifies a $C^{1}$ Lyapunov function to prove uniform asymptotic stability as well as uniform finite time stability in the presence of a class of disturbances that have non-Lipschitz upper bound.
\end{abstract}

\section{INTRODUCTION}

Finite time stabilisation has been an active area of research in control systems engineering. The finite time convergence property of the closed-loop system inherently requires the dynamics to be governed by a non-Lipschitz right hand side [1]. The main motivation to study finite time controllers and finite time stability is in its superior robustness properties [2] while giving better precision in tracking problems. Work on continuous finite time controllers can be found in [3] and [4] for the double integrator system. Subsequently, there have been many results in both the theory and application of finite time controllers. An output feedback based finite time controller can be found in [5]. Finite time stability of a class of time invariant continuous systems can be found in [6].

Amongst various routes to proving asymptotic stability, the homogeneity of the right hand side of differential equations has been a mathematically sound and proven technique [7], [8]. It is also known that homogeneity leads to finite time stability of the system when the degree of homogeneity is negative and the system is asymptotically stable [9]. A similar result can be found in [10] where finite time stability of discontinuous non-autonomous systems was proved when the system is uniformly asymptotically stable and the degree of homogeneity is negative. Uniform finite time stability of continuous non-autonomous systems has been established in [11].

There have been many advances in terms of studying robust finite time controllers. The homogeneous domination approach developed in [12] studies robustness of homogeneous controllers to non-linear time varying disturbance

This work was supported by EPSRC via research grant EP/J018295/1.

H. B. Oza and S. K. Spurgeon are with School of Engineering and Digital Arts, University of Kent, Canterbury, Kent CT2 7NT, UK; H.B.Oza@kent.ac.uk, S.K.Spurgeon@kent.ac.uk

Yury V. Orlov is also with CICESE Research Center, Ensenada, Mexico yorlov@cicese.mx functions. Finite time stability using the concept of so-called homogeneity in the bi-limit was established in [13, Corollary 2.24]. Reference [14] proves finite time stability of a class of time varying non-linear systems. A recent advance for the finite time stabilisation of a double integrator system can be found in [15] where finite time output feedback was studied without considering robustness to disturbances. Reference [16] proposes a Lyapunov function for the perturbed double integrator, however, the robustness claims are presented without proof. Reference [17] proves that an augmented continuous sliding mode controller is robust to persisting disturbances but with the trade-off that the derivative of the disturbance is required to be bounded.

Since finite time stable continuous autonomous systems are known to preserve finite time stability even in the presence of disturbance growing linearly with state [2, Theorem 5.3] and since it is also known that continuous autonomous finite time stable systems have ultimately bounded trajectories for persisting disturbances [2, Theorem 5.2], it is reasonable to expect finite time controllers to be able to reject disturbances which admit non-Lipschitz upper bound vanishing in the origin. This paper proves that such intuitive expectation holds true for the class of homogeneous finite time controllers [3, Section 4] when a class of time varying continuous disturbances admitting a non-Lipschitz upper bound affect the double integrator system.

The paper is organized as follows. Section II summarizes various definitions underpinning the subsequent sections and presents the problem formulation. The main result of proving robustness to purely continuous disturbances is presented in Section III. It should be noted that the main result of Section III presents a detailed proof of a special case of one of the claims being reported without proof in the reference [18] which studies robustness of continuous finite time controllers in the presence of a broader class of discontinuous time varying disturbances. Section IV presents conclusion.

\section{PReliminaries}

This section collects important definitions and results from the literature which are utilised throughout the paper. Consider the dynamical system

$$
\dot{x}=\phi(x, t)
$$

where $x=\left(x_{1}, x_{2}, \ldots, x_{n}\right)^{\mathrm{T}}$ is the state vector, $t \in \mathbf{R}$ is the time variable and function $\phi(x, t)$ is a continuous function of state and time. The paper mainly focusses on uncertain systems of the type

$$
\dot{x}=\phi(x, t)+\psi(x, t)
$$


where $\psi(x, t)$ is an uncertain continuous function of state and time. It is assumed that disturbance $\psi(x, t)$ admits an upper bound that vanishes in the origin, i.e.

$$
\sup _{t \geq 0} \lim _{x \rightarrow 0}|\psi(x, t)| \rightarrow 0 \text {. }
$$

The paper deals with non-Lipschitz continuous right hand sides. The solutions of the resulting differential equations are non-unique in general. See [19, Section 10], [2, Section 2] and references therein for conditions of uniqueness of solutions in forward and reverse time for systems governed by non-Lipschitz continuous right hand sides. Following definitions pertaining to various types of stability are revised from [10] to suit the present case of continuous right hand side.

Definition 1 (Equiuniform stability [10]): The

equilibrium point $x=0$ of the uncertain system (2), (3) is equiuniformly stable iff for each $t_{0} \in \mathbf{R}, \varepsilon>0$ there exists $\delta=\delta(\varepsilon)$, dependent on $\varepsilon$ and independent of $t_{0}$ and $\psi$, such that each solution $x\left(\cdot, t_{0}, x^{0}\right)$ of (2), (3) with the initial data $x^{0} \in B_{\delta}$; where $B_{\delta}$ is a ball of radius $\delta$, exists on the semi-infinite time interval $\left[t_{0}, \infty\right)$ and satisfies the inequality

$$
\left\|\left(x\left(t, t_{0}, x^{0}\right)\right)\right\| \leq \varepsilon \quad \forall t \in\left[t_{0}, \infty\right) .
$$

Definition 2 (Equiuniform asymptotic stability [10]):

The equilibrium point $x=0$ of the uncertain system (2), (3) is said to be equiuniformly asymptotically stable if it is equiuniformly stable and the convergence

$$
\lim _{t \rightarrow \infty}\left\|\left(x\left(t, t_{0}, x^{0}\right)\right)\right\| \rightarrow 0
$$

holds for all the solutions $x\left(\cdot, t_{0}, x^{0}\right)$ of the uncertain system (2), (3) initialized within some $B_{\delta}$ (uniformly in the initial data $t_{0}$ and $x^{0}$ ). If this convergence remains in force for each $\delta>0$, the equilibrium point is said to be globally equiuniformly asymptotically stable.

Definition 3 (Equiuniform finite time stability [10]): The equilibrium point $x=0$ of the uncertain system (2), (3) is said to be globally equiuniformly finite time stable if, in addition to the global equiuniform asymptotical stability, the limiting relation

$$
x\left(t, t_{0}, x^{0}\right)=0
$$

holds for all the solutions $x\left(\cdot, t_{0}, x^{0}\right)$ and for all $t \geq t_{0}+$ $T\left(t_{0}, x^{0}\right)$, where the settling time function

$$
T\left(t_{0}, x^{0}\right)=\sup _{x\left(\cdot, t_{0}, x^{0}\right)} \inf \left\{T \geq 0: x\left(t, t_{0}, x^{0}\right)=0 \quad \forall t \geq t_{0}+T\right\}
$$

is such that

$$
T\left(B_{\delta}\right)=\sup _{x^{0} \in B_{\delta}, t_{0} \in \mathbf{R}} T\left(t_{0}, x^{0}\right)<\infty \quad \forall \delta>0,
$$

where $\delta=\delta(\varepsilon)$ is independent of $t_{0}$ and $\psi$.

Definition 4: (Homogeneity of differential equations [10], [20], [7]) The differential equation (1) (or the uncertain system (2), (3)) is called homogeneous of degree $q \in \mathbf{R}$ with respect to dilation $\left(r_{1}, r_{2}, \ldots, r_{n}\right)$, where $r_{i}>0, i=1,2, \ldots, n$, if there exists a constant $c_{0}>0$, called a lower estimate of the homogeneity parameter such that any solution $x(\cdot)$ of (1) (respectively, that of the uncertain system (2), (3)) generates a parameterized set of solutions $x^{c}(\cdot)$ with components

$$
x_{i}^{c}(t)=c^{r_{i}} x_{i}\left(c^{q} t\right)
$$

and any parameter $c \geq c_{0}$.

The following definition is a special case of the definition of homogeneous piece-wise continuous functions [10, Definition 2.10].

Definition 5: A continuous function $\phi: \mathbf{R}^{n+1} \rightarrow \mathbf{R}^{n}$ is called homogeneous of degree $q \in \mathbf{R}$ with respect to dilation $\left(r_{1}, r_{2}, \ldots, r_{n}\right)$, where $r_{i}>0, i=1,2, \ldots, n$, if there exists a constant $c_{0}>0$ such that

$$
\phi_{i}\left(c^{r_{1}} x_{1}, c^{r_{2}} x_{2}, \ldots, c^{r_{n}} x_{n}, c^{-q t}\right)=c^{q+r_{i}} \phi_{i}\left(x_{1}, x_{2}, \ldots, x_{n}, t\right)
$$

for all $c \geq c_{0}$.

\section{A. Problem Statement}

Consider the following perturbed double integrator:

$$
\dot{x}_{1}=x_{2} \quad \dot{x}_{2}=u\left(x_{1}, x_{2}\right)+\omega(x, t)
$$

where $x=\left(x_{1}, x_{2}\right)^{T} \in \mathbf{R}^{2}$ is the state vector, $u$ is the control input, $\omega(x, t)$ is a continuous disturbance. Consider the following homogeneous controller proposed in [3]:

$$
u\left(x_{1}, x_{2}\right)=-\mu_{1}\left|x_{2}\right|^{\alpha} \operatorname{sign}\left(x_{2}\right)-\mu_{2}\left|x_{1}\right|^{\frac{\alpha}{2-\alpha}} \operatorname{sign}\left(x_{1}\right),
$$

where $\alpha \in(0,1)$ is a scalar and $\mu_{1}, \mu_{2}$ are controller gains.

Assumption 1: The disturbance $\omega(x, t)$ is assumed to admit a uniform non-Lipschitz upper bound as follows:

$$
\sup _{t \geq 0}\left|\omega\left(x_{1}, x_{2}, t\right)\right| \leq M\left|x_{2}\right|^{\alpha}
$$

The aim of the paper is to establish equiuniform finite-time stability of the uncertain system (11), (12) in the presence of disturbances admitting the upper bound (13).

\section{LYAPUNOV ANALYSIS}

As mentioned in Section I, this section presents the main result of the paper by giving a detailed proof of one of the theorems being reported in [18] without proof. Equiuniform finite time stability of the closed-loop system (11), (12) is proved in Theorem 1. The following instrumental lemma is extracted from [18] for later use:

Lemma 1: Let the function $\omega\left(x_{1}, x_{2}, t\right)$ be a continuous function which is uniformly bounded by the upper-bound (13). Then, the uncertain differential equation (11),(12) with the uncertainty constraints (13) is homogeneous of degree $q=-1$ with respect to the dilation $\left(r_{1}, r_{2}\right)=\left(\frac{2-\alpha}{1-\alpha}, \frac{1}{1-\alpha}\right)$.

Proof: Let $x(\cdot)=\left(x_{1}(\cdot), x_{2}(\cdot)\right)^{T}$ be a solution of $(11),(12)$ under some continuous function $\omega(x, t)$, satisfying (13). Then it is straightforward to verify that for arbitrary $c \geq \max \left(1, c_{0}\right)$ the function $x^{c}(\cdot)$ with components $x_{i}^{c}(t)=$ $c^{r_{i}} x_{i}\left(c^{q} t\right), i=1,2$, is a solution of $(11),(12)$ with the continuous function $\omega\left(x_{1}, x_{2}, t\right) \triangleq \omega^{c}\left(x_{1}, x_{2}, t\right)$ which is as follows:

$$
\omega^{c}\left(x_{1}, x_{2}, t\right)=c^{q+r_{2}} \omega\left(c^{-r_{1}} x_{1}, c^{-r_{2}} x_{2}, c^{q} t\right)
$$


where, the right hand side represents a parameterized set of uncertainties. The following holds true due to the parameterisation (14):

$$
\begin{aligned}
\left|\omega^{c}\left(x_{1}, x_{2}, t\right)\right| & =\left|c^{q+r_{2}} \omega\left(c^{-r_{1}} x_{1}, c^{-r_{2}} x_{2}, c^{q} t\right)\right| \\
\Rightarrow \quad\left|\omega^{c}\left(x_{1}, x_{2}, t\right)\right| & \leq c^{q+r_{2}} M\left|c^{-r_{2}} x_{2}\right|^{\alpha} \leq c^{q+r_{2}-\alpha r_{2}} M\left|x_{2}\right|^{\alpha}
\end{aligned}
$$

Hence, all parameterized disturbance functions represented by the right hand side of (14) are admissible in the sense of (13) if the following holds true:

$$
c^{q+r_{2}-\alpha r_{2}} \leq 1
$$

From the definitions $r_{2}=\frac{1}{1-\alpha}, q=-1$, it is obtained that

$$
q+r_{2}-\alpha r_{2}=0 \Rightarrow c^{q+r_{2}-\alpha r_{2}} \leq 1
$$

and that the function $\omega^{c}\left(x_{1}, x_{2}, t\right)$ is admissible in the sense of (13). Recalling Definitions 4, 5 and Lemma [10, Lemma 2.11], the solutions $x_{1}^{c}(t)=c^{r_{1}} x_{1}\left(c^{q} t\right), x_{2}^{c}(t)=c^{r_{2}} x_{2}\left(c^{q} t\right)$ are solutions of the system (11), (12) with the continuous function $\omega^{c}\left(x_{1}, x_{2}, t\right)$ given by (14). Thus, any solution of the differential equation (11), (12) generates a parameterized set of solutions $x_{1}^{c}(t), x_{2}^{c}(t)$ with the parameter $c$ large enough. Hence, (11), (12) is homogeneous of degree $q=-1$ with the dilation $\left(r_{1}, r_{2}\right)=\left(\frac{2-\alpha}{1-\alpha}, \frac{1}{1-\alpha}\right)$. This proves the statement of Lemma 1.

The proof presented above is a special case of that being reported in [18] suited to the present case of continuous disturbances.

Theorem 1: Given $\alpha \in\left(\frac{2}{3}, 1\right)$, the closed-loop system (11), (12) is globally equiuniformly finite time stable, regardless of whichever disturbance $\omega(x, t)$, satisfying condition (13) with $0<M<\mu_{1}<\mu_{2}-M$, affects the system.

Proof: The proof is given in several steps.

1. Global Asymptotic Stability Let the following candidate Lyapunov function $V$ be considered [3], [21]:

$$
V\left(x_{1}, x_{2}\right)=\mu_{2} \frac{2-\alpha}{2}\left|x_{1}\right|^{\frac{2}{2-\alpha}}+\frac{1}{2} x_{2}^{2}
$$

Under the conditions of the theorem, the time derivative of the function $V\left(x_{1}, x_{2}\right)$, computed along the trajectories of (11), (12) is estimated as follows [21, Th. 1]:

$$
\dot{V} \leq-\left(\mu_{1}-M\right)\left|x_{2}\right|^{\alpha+1}
$$

Noting that $M<\mu_{1}$ by a condition of the theorem and that the equilibrium point $x_{1}=x_{2}=0$ is the only trajectory of (11), (12) on the invariance manifold $x_{2}=0$ where $\dot{V}\left(x_{1}, x_{2}\right)=0$, the global asymptotic stability of (11), (12) is then established by applying the invariance principle [22], [23].

\section{Semiglobal Strong Lyapunov Functions.}

This step shows the existence of a parameterized family of semi-global Lyapunov functions $V_{\tilde{R}}\left(x_{1}, x_{2}\right)$, with an a priori but arbitrarily given $\tilde{R}>0$, such that each $V_{\tilde{R}}\left(x_{1}, x_{2}\right)$ is wellposed on the corresponding compact set

$$
D_{\tilde{R}}=\left\{\left(x_{1}, x_{2}\right) \in \mathbf{R}^{2}: V\left(x_{1}, x_{2}\right) \leq \tilde{R}\right\} .
$$

In other words, $V_{\tilde{R}}\left(x_{1}, x_{2}\right)$ is to be positive definite on $D_{\tilde{R}}$ and its derivative, computed along the trajectories of the uncertain system (11), (12) with initial conditions within $D_{\tilde{R}}$, is to be negative definite in the sense that,

$$
\dot{V}_{\tilde{R}}\left(x_{1}, x_{2}\right) \leq-W_{\tilde{R}}\left(x_{1}, x_{2}\right)
$$

for all $\left(x_{1}, x_{2}\right) \in D_{\tilde{R}}$ and for some $W_{\tilde{R}}\left(x_{1}, x_{2}\right)$, positive definite on $D_{\tilde{R}}$. A parameterized family of Lyapunov functions $V_{\tilde{R}}\left(x_{1}, x_{2}\right), \tilde{R}>0$, with the properties defined above are constructed by combining the Lyapunov function $V$ of (18), whose time derivative along the system motion is only negative semi-definite, with the indefinite functions

$$
\begin{aligned}
U\left(x_{1}, x_{2}\right) & =U_{1}\left(x_{1}, x_{2}\right)+U_{2}\left(x_{1}, x_{2}\right)+U_{3}\left(x_{1}, x_{2}\right) \\
U_{1}\left(x_{1}, x_{2}\right) & =\kappa_{1}\left|x_{1}\right|^{\frac{2 \alpha}{2-\alpha}} \operatorname{sign}\left(x_{1}\right)\left|x_{2}\right|^{2 \alpha} x_{2} \\
U_{2}\left(x_{1}, x_{2}\right) & =\kappa_{1} \kappa_{2} x_{1}^{3} x_{2}\left|x_{2}\right|^{\alpha}, U_{3}\left(x_{1}, x_{2}\right)=\kappa_{1} \kappa_{2} \kappa_{3} x_{1}^{5} x_{2}
\end{aligned}
$$

as follows:

$$
V_{\tilde{R}}\left(x_{1}, x_{2}\right)=V\left(x_{1}, x_{2}\right)+\sum_{i=1}^{3} U_{i}\left(x_{1}, x_{2}\right)
$$

where the positive weight scalars $\kappa_{i}, i=1,2,3$ are chosen small enough so that,

$$
\begin{aligned}
& \kappa_{2}<\frac{(1+2 \alpha) \mu_{2}}{(1+\alpha)\left(\mu_{1}+M\right) \rho^{3(1-\alpha)}}, \rho=\frac{2 \tilde{R}}{\mu_{2}(2-\alpha)}, \\
& \kappa_{3}<\frac{(1+\alpha) \mu_{2}}{\left(\mu_{1}+M\right) \rho^{\frac{4-3 \alpha}{2}}} \\
& \kappa_{1}<\min \left\{\begin{array}{l}
\frac{\mu_{1}-M}{K_{1}}, \frac{\mu_{2}(2-\alpha)}{\kappa_{2} \rho^{5-3 \alpha}\left(1+\kappa_{3} \rho^{2(2-\alpha)}\right)}, \\
\frac{1}{\rho^{2 \alpha}+(2 \tilde{R})^{2 \alpha-1}+\kappa_{2}\left((2 \tilde{R})^{\alpha}+\kappa_{3}\right)}
\end{array}\right\} \text {, } \\
& K_{1}=\frac{2 \alpha}{2-\alpha} \rho^{\frac{3 \alpha-2}{2}}(2 \tilde{R})^{\frac{1+\alpha}{2}}+\left(\mu_{1}+M\right)(1+2 \alpha) \rho^{\alpha}(2 \tilde{R})^{\frac{2 \alpha-1}{2}} \\
& +3 \kappa_{2} \rho^{2-\alpha}(2 \tilde{R})^{\frac{1}{2}}+5 \kappa_{2} \kappa_{3} \rho^{2(2-\alpha)}(2 \tilde{R})^{\frac{1-\alpha}{2}} .
\end{aligned}
$$

An a priori definition of the scalars $\kappa_{i}$ is always possible. This is because for known initial conditions $x^{0} \in \mathbf{R}^{2}$, a given bound $M$ and in fixed values of $\mu_{1}, \mu_{2}$, there always exists an arbitrarily large $\tilde{R}$ such that $V \leq \tilde{R}$ holds true. In the first step, $\kappa_{2}$ and $\kappa_{3}$ can be computed using $\rho$ and (24). In the second step, the constant $K_{1}$ can be computed using $\kappa_{2}, \kappa_{3}$ of the first step and using definition (25). In the final step, $\kappa_{1}$ can be computed using $\kappa_{2}, \kappa_{3}, K_{1}$ of previous steps and (24). Hence, (24), (25) define all $\kappa_{i}, i=1,2.3$ unambiguously.

Remark 1: The functions $V_{\tilde{R}}$ and $U_{i}, i=1,2,3$ are not only continuous but also $C^{1}$ smooth for all $x \in \mathbf{R}^{2}$ for $\alpha \in\left(\frac{2}{3}, 1\right)$. Setting $\alpha=0$ in the following analysis corresponds to the discontinuous case for which the finite time stability has been established following a similar semi-global analysis $[10, \mathrm{Th}$ 4.2]. It is further noted that the expressions $2 \alpha-1>0,3 \alpha-$ $2>0$ hold true due to $\alpha \in\left(\frac{2}{3}, 1\right)$ in the derivations below.

Due to (19), all possible solutions of the uncertain system (11), (12), initialized at $t_{0} \in \mathbf{R}$ within the compact set (20), are a priori estimated by

$$
\sup _{t \in\left[t_{0}, \infty\right)} V\left(x_{1}, x_{2}\right) \leq \tilde{R} .
$$


The following inequalities hold true:

$$
\left|x_{1}\right|^{\frac{2}{2-\alpha}} \leq \rho, \quad\left|x_{2}\right| \leq \sqrt{2 \tilde{R}} .
$$

Let the positive definiteness of the Lyapunov function (23) be verified. The following analysis is in order for the indefinite functions $U_{i}, i=1,2,3$.

$$
\begin{aligned}
U_{1}\left(x_{1}, x_{2}\right) & =\kappa_{1}\left|x_{1}\right|^{\frac{2 \alpha}{2-\alpha}} \operatorname{sign}\left(x_{1}\right)\left|x_{2}\right|^{2 \alpha} x_{2} \\
& \geq-\frac{\kappa_{1}}{2}\left|x_{1}\right|^{\frac{4 \alpha}{2-\alpha}} x_{2}^{2}-\frac{\kappa_{1}}{2}\left|x_{2}\right|^{4 \alpha} \\
& \geq-\left(\rho^{2 \alpha}+(2 \tilde{R})^{2 \alpha-1}\right) \frac{\kappa_{1}}{2} x_{2}^{2}
\end{aligned}
$$

where, (27) and the trivial inequality $2 a b>-\left(a^{2}+\right.$ $\left.b^{2}\right), \forall a, b \in R$ have been utilised. Similarly, $U_{2}$ and $U_{3}$ can also be analysed as follows:

$$
\begin{aligned}
U_{2}\left(x_{1}, x_{2}\right) & =\kappa_{1} \kappa_{2} x_{1}^{3} x_{2}\left|x_{2}\right|^{\alpha} \\
& \geq-\frac{\kappa_{1} \kappa_{2}}{2}\left(x_{1}^{6}+x_{2}^{2}\left|x_{2}\right|^{2 \alpha}\right) \\
& \geq-\frac{\kappa_{1} \kappa_{2}}{2}\left|x_{1}\right|^{\frac{2}{2-\alpha}} \rho^{5-3 \alpha}-\frac{\kappa_{1} \kappa_{2}}{2}(2 \tilde{R})^{\alpha} x_{2}^{2} \\
U_{3}\left(x_{1}, x_{2}\right) & =\kappa_{1} \kappa_{2} \kappa_{3} x_{1}^{5} x_{2} \\
& \geq-\frac{\kappa_{1} \kappa_{2} \kappa_{3}}{2} x_{1}^{10}-\kappa_{1} \kappa_{2} \kappa_{3} x_{2}^{2} \\
& \geq-\frac{\kappa_{1} \kappa_{2} \kappa_{3}}{2}\left|x_{1}\right|^{\frac{2}{2-\alpha}} \rho^{9-5 \alpha}-\frac{\kappa_{1} \kappa_{2} \kappa_{3}}{2} x_{2}^{2}
\end{aligned}
$$

Hence, the Lyapunov function (23) is positive definite on compacta (20); for all $\left(x_{1}, x_{2}\right) \in D_{\tilde{R}} \backslash\{0,0\}$ and $\kappa_{i}>0, i=$ 1,2,3 satisfying (24), as shown below:

$$
\begin{aligned}
& V_{\tilde{R}}\left(x_{1}, x_{2}\right)=\mu_{2} \frac{2-\alpha}{2}\left|x_{1}\right|^{\frac{2}{2-\alpha}}+\frac{1}{2} x_{2}^{2}+\sum_{i=1}^{3} U_{i}\left(x_{1}, x_{2}\right) \\
& \geq\left(\mu_{2} \frac{2-\alpha}{2}-\frac{\kappa_{1} \kappa_{2}}{2} \rho^{5-3 \alpha}\left(1+\kappa_{3} \rho^{2(2-\alpha)}\right)\right)\left|x_{1}\right|^{\frac{2}{2-\alpha}} \\
& +\left(1-\kappa_{1}\left(\rho^{2 \alpha}+(2 \tilde{R})^{2 \alpha-1}+\kappa_{2}\left((2 \tilde{R})^{\alpha}+\kappa_{3}\right)\right)\right) \frac{1}{2} x_{2}^{2} \\
& \geq L_{\tilde{R}} V\left(x_{1}, x_{2}\right)
\end{aligned}
$$

where inequalities (28), (29) and (30) are utilised and

$$
\begin{aligned}
L_{\tilde{R}} & <\min \left\{L_{\tilde{R}_{1}}, L_{\tilde{R}_{2}}\right\} \\
L_{\tilde{R}_{1}} & =1-\kappa_{1}\left(\rho^{2 \alpha}+(2 \tilde{R})^{2 \alpha-1}\right)-\kappa_{1} \kappa_{2}\left((2 \tilde{R})^{\alpha}\right)-\kappa_{1} \kappa_{2} \kappa_{3} \\
L_{\tilde{R}_{2}} & =\frac{\mu_{2}(2-\alpha)}{2}-\frac{\kappa_{1} \kappa_{2} \rho^{5-3 \alpha}}{2}\left(1+\kappa_{3} \rho^{2(2-\alpha)}\right)
\end{aligned}
$$

It should be noted that $L_{\tilde{R}_{1}}>0, L_{\tilde{R}_{2}}>0, L_{\tilde{R}}>0$ due to (24) and hence positive definiteness of $V_{\tilde{R}}$ is ensured from (31) on $D_{\tilde{R}} \backslash\{0,0\}$. Similarly, it can be shown that the following inequality holds true:

$$
\begin{aligned}
& V_{\tilde{R}}\left(x_{1}, x_{2}\right)=\mu_{2} \frac{2-\alpha}{2}\left|x_{1}\right|^{\frac{2}{2-\alpha}}+\frac{1}{2} x_{2}^{2}+\sum_{i=1}^{3} U_{i}\left(x_{1}, x_{2}\right) \\
& \leq\left(\mu_{2} \frac{2-\alpha}{2}+\frac{\kappa_{1} \kappa_{2}}{2} \rho^{5-3 \alpha}\left(1+\kappa_{3} \rho^{2(2-\alpha)}\right)\right)\left|x_{1}\right|^{\frac{2}{2-\alpha}} \\
& +\left(1+\kappa_{1}\left(\rho^{2 \alpha}+(2 \tilde{R})^{2 \alpha-1}+\kappa_{2}\left((2 \tilde{R})^{\alpha}+\kappa_{3}\right)\right)\right) \frac{1}{2} x_{2}^{2} \\
& \leq M_{\tilde{R}} V\left(x_{1}, x_{2}\right)
\end{aligned}
$$

where, the trivial inequality $2 a b<\left(a^{2}+b^{2}\right), \forall a, b \in R$ is used and

$$
\begin{aligned}
M_{\tilde{R}}>\max \left\{M_{\tilde{R}_{1}}, M_{\tilde{R}_{2}}\right\}, \text { where } \\
M_{\tilde{R}_{1}}=\mu_{2} \frac{2-\alpha}{2}+\frac{\kappa_{1} \kappa_{2}}{2} \rho^{5-3 \alpha}\left(1+\kappa_{3} \rho^{2(2-\alpha)}\right) \\
M_{\tilde{R}_{2}}=1+\kappa_{1}\left(\rho^{2 \alpha}+(2 \tilde{R})^{2 \alpha-1}\right)+\kappa_{1} \kappa_{2}\left((2 \tilde{R})^{\alpha}\right)+\kappa_{1} \kappa_{2} \kappa_{3},
\end{aligned}
$$

is a positive scalar. The time derivative of the indefinite function $U_{1}\left(x_{1}, x_{2}\right)$ along the trajectories of the uncertain system (11), (12) is as follows:

$$
\begin{aligned}
\dot{U}_{1}\left(x_{1}, x_{2}\right)=\kappa_{1} \frac{2 \alpha}{2-\alpha}\left|x_{1}\right|^{\frac{3 \alpha-2}{2-\alpha}}\left|x_{2}\right|^{2 \alpha+2} \\
\quad+\kappa_{1}(1+2 \alpha)\left|x_{1}\right|^{\frac{2 \alpha}{2-\alpha}} \operatorname{sign}\left(x_{1}\right)\left|x_{2}\right|^{2 \alpha} \dot{x}_{2} \\
=\kappa_{1} \frac{2 \alpha}{2-\alpha}\left|x_{1}\right|^{\frac{3 \alpha-2}{2-\alpha}}\left|x_{2}\right|^{2 \alpha+2} \\
\quad-\kappa_{1} \mu_{1}\left|x_{1}\right|^{\frac{2 \alpha}{2-\alpha}} \operatorname{sign}\left(x_{1}\right)\left|x_{2}\right|^{3 \alpha} \operatorname{sign}\left(x_{2}\right) \\
-\kappa_{1} \mu_{2}\left|x_{1}\right|^{\frac{3 \alpha}{2-\alpha}}\left|x_{2}\right|^{2 \alpha}+\kappa_{1} \omega\left|x_{1}\right|^{\frac{2 \alpha}{2-\alpha}} \operatorname{sign}\left(x_{1}\right)\left|x_{2}\right|^{2 \alpha} \\
\quad-2 \alpha \kappa_{1} \mu_{1}\left|x_{1}\right|^{\frac{2 \alpha}{2-\alpha}} \operatorname{sign}\left(x_{1}\right)\left|x_{2}\right|^{3 \alpha} \operatorname{sign}\left(x_{2}\right) \\
\quad-2 \alpha \kappa_{1} \mu_{2}\left|x_{1}\right|^{\frac{3 \alpha}{2-\alpha}}\left|x_{2}\right|^{2 \alpha} \\
\quad+2 \alpha \kappa_{1} \omega\left|x_{1}\right|^{\frac{2 \alpha}{2-\alpha}} \operatorname{sign}\left(x_{1}\right)\left|x_{2}\right|^{2 \alpha} \\
\leq-\kappa_{1} \mu_{2}(1+2 \alpha)\left|x_{1}\right|^{\frac{3 \alpha}{2-\alpha}}\left|x_{2}\right|^{2 \alpha}+\kappa_{1} \frac{2 \alpha}{2-\alpha}\left|x_{1}\right|^{\frac{3 \alpha-2}{2-\alpha}}\left|x_{2}\right|^{2 \alpha+2} \\
\quad+\kappa_{1}\left(\mu_{1}+M\right)(1+2 \alpha)\left|x_{1}\right|^{\frac{2 \alpha}{2-\alpha}}\left|x_{2}\right|^{3 \alpha}
\end{aligned}
$$

The temporal derivative of $U_{2}$ along the trajectories of the closed-loop system (11), (12) is as follows:

$$
\begin{aligned}
\dot{U}_{2}= & 3 \kappa_{1} \kappa_{2} x_{1}^{2}\left|x_{2}\right|^{\alpha+2}+\kappa_{1} \kappa_{2}(1+\alpha) x_{1}^{3}\left|x_{2}\right|^{\alpha} \dot{x}_{2} \\
= & 3 \kappa_{1} \kappa_{2} x_{1}^{2}\left|x_{2}\right|^{\alpha+2}-\kappa_{1} \kappa_{2} \mu_{1} x_{1}^{3}\left|x_{2}\right|^{2 \alpha} \operatorname{sign}\left(x_{2}\right) \\
& +\kappa_{1} \kappa_{2} x_{1}^{3}\left|x_{2}\right|^{\alpha} \omega-\kappa_{1} \kappa_{2} \mu_{2} x_{1}^{2}\left|x_{1}\right|^{\frac{2}{2-\alpha}}\left|x_{2}\right|^{\alpha} \\
& -\alpha \kappa_{1} \kappa_{2} \mu_{1} x_{1}^{3}\left|x_{2}\right|^{2 \alpha} \operatorname{sign}\left(x_{2}\right) \\
& +\alpha \kappa_{1} \kappa_{2} x_{1}^{3}\left|x_{2}\right|^{\alpha} \omega-\alpha \kappa_{1} \kappa_{2} \mu_{2} x_{1}^{2}\left|x_{1}\right|^{\frac{2}{2-\alpha}}\left|x_{2}\right|^{\alpha} \\
\leq & 3 \kappa_{1} \kappa_{2} x_{1}^{2}\left|x_{2}\right|^{\alpha+2}-\kappa_{1} \kappa_{2}(1+\alpha) \mu_{2} x_{1}^{2}\left|x_{1}\right|^{\frac{2}{2-\alpha}}\left|x_{2}\right|^{\alpha} \\
& +\kappa_{1} \kappa_{2}(1+\alpha)\left(\mu_{1}+M\right)\left|x_{1}\right|^{3}\left|x_{2}\right|^{2 \alpha}
\end{aligned}
$$

The temporal derivative of $U_{3}$ along the trajectories of the closed-loop system (11), (12) can be obtained as follows:

$$
\begin{aligned}
\dot{U}_{3}= & 5 \kappa_{1} \kappa_{2} \kappa_{3} x_{1}^{4} x_{2}^{2}+\kappa_{1} \kappa_{2} \kappa_{3} x_{1}^{5} \dot{x}_{2} \\
= & 5 \kappa_{1} \kappa_{2} \kappa_{3} x_{1}^{4} x_{2}^{2}-\kappa_{1} \kappa_{2} \kappa_{3} \mu_{1} x_{1}^{5}\left|x_{2}\right|^{\alpha} \operatorname{sign}\left(x_{2}\right) \\
& \quad-\kappa_{1} \kappa_{2} \kappa_{3} \mu_{2} x_{1}^{4}\left|x_{1}\right|^{\frac{2}{2-\alpha}}+\kappa_{1} \kappa_{2} \kappa_{3} x_{1}^{5} \omega \quad(37) \\
\leq & 5 \kappa_{1} \kappa_{2} \kappa_{3} x_{1}^{4} x_{2}^{2}+\kappa_{1} \kappa_{2} \kappa_{3}\left(\mu_{1}+M\right)\left|x_{1}\right|^{5}\left|x_{2}\right|^{\alpha} \\
& -\kappa_{1} \kappa_{2} \kappa_{3} \mu_{2} x_{1}^{4}\left|x_{1}\right|^{\frac{2}{2-\alpha}}
\end{aligned}
$$

It should be noted that the inequality

$$
\left|x_{2}\right|^{2 \alpha}=\left|x_{2}\right|\left|x_{2}\right|^{2 \alpha-1} \leq\left|x_{2}\right|(2 \tilde{R})^{\frac{2 \alpha-1}{2}}
$$

holds true due to the condition $\alpha \in\left(\frac{2}{3}, 1\right)$ of the theorem. The last inequalities of (35), (36) and (37) are re-written by 
utilising (27) and (38) as follows:

$$
\begin{gathered}
\sum_{i=1}^{3} \dot{U}_{i}\left(x_{1}, x_{2}\right) \leq-\beta_{1} x_{1}^{2}\left|x_{1}\right|^{\frac{2}{2-\alpha}}\left|x_{2}\right|^{\alpha}-\beta_{2}\left|x_{1}\right|^{\frac{3 \alpha}{2-\alpha}}\left|x_{2}\right|^{2 \alpha} \\
+\kappa_{1} K_{1}\left|x_{2}\right|^{\alpha+1}
\end{gathered}
$$

where,

$$
\begin{aligned}
& \beta_{1}=\kappa_{1} \kappa_{2}\left((1+\alpha) \mu_{2}-\kappa_{3}\left(\mu_{1}+M\right) \rho^{\frac{4-3 \alpha}{2}}\right) \\
& \beta_{2}=\kappa_{1}\left(\mu_{2}(1+2 \alpha)-\kappa_{2}(1+\alpha)\left(\mu_{1}+M\right) \rho^{3(1-\alpha)}\right),
\end{aligned}
$$

$K_{1}>0$ from (24) and the corresponding upper bound on $\left|x_{1}\right|$ and $\left|x_{2}\right|$ from (27) are utilised. It should be noted that $\kappa_{i}, i=1,2,3$ are all positive constants due to (24). Hence, by combining (19) and (39), the time derivative of (23) can be obtained as follows:

$$
\begin{aligned}
\dot{V}_{\tilde{R}} & \leq-\beta_{1} x_{1}^{2}\left|x_{1}\right|^{\frac{2}{2-\alpha}}\left|x_{2}\right|^{\alpha}-\beta_{2}\left|x_{1}\right|^{\frac{3 \alpha}{2-\alpha}}\left|x_{2}\right|^{2 \alpha} \\
& -\left(\mu_{1}-M-\kappa_{1} K_{1}\right)\left|x_{2}\right|^{\alpha+1}-\kappa_{1} \kappa_{2} \kappa_{3} \mu_{2} x_{1}^{4}\left|x_{1}\right|^{\frac{2}{2-\alpha}},
\end{aligned}
$$

It should be noted that the expressions $\beta_{1}>0, \beta_{2}>0$ hold true due to (24). Ignoring the negative semi-definite terms with $\beta_{1}, \beta_{2}$, (41) can be rewritten as follows:

$\dot{V}_{\tilde{R}} \quad \leq-\left(\mu_{1}-M-\kappa_{1} K_{1}\right)\left|x_{2}\right|^{\alpha+1}-\kappa_{1} \kappa_{2} \kappa_{3} \mu_{2} x_{1}^{4}\left|x_{1}\right|^{\frac{2}{2-\alpha}}$

Furthermore, the following inequalities hold true within the compacta (20):

$$
\begin{aligned}
& x_{2}^{2}=\left|x_{2}\right|^{2}=\left|x_{2}\right|^{\alpha+1}\left|x_{2}\right|^{1-\alpha} \leq\left|x_{2}\right|^{\alpha+1}(\sqrt{2 \tilde{R}})^{1-\alpha} \\
& \Rightarrow \quad-\left|x_{2}\right|^{\alpha+1} \leq-\frac{x_{2}^{2}}{(\sqrt{2 \tilde{R}})^{1-\alpha}}
\end{aligned}
$$

Hence, (42) can be simplified as follows:

$$
\dot{V}_{\tilde{R}} \leq-c_{\tilde{R}}\left(\left|x_{1}\right|^{\frac{10-4 \alpha}{2-\alpha}}+x_{2}^{2}\right)
$$

where,

$$
c_{\tilde{R}}=\min \left\{\frac{\mu_{1}-M-\kappa_{1} K_{1}}{(\sqrt{2 \tilde{R}})^{1-\alpha}}, \quad \kappa_{1} \kappa_{2} \kappa_{3} \mu_{2}\right\}>0 .
$$

Case 1: $\left|x_{1}\right| \geq 1$ :

The following inequality holds true for $\left|x_{1}\right| \geq 1$ :

$$
\frac{10-4 \alpha}{2-\alpha} \geq \frac{2}{2-\alpha} \Leftrightarrow\left|x_{1}\right|^{\frac{10-4 \alpha}{2-\alpha}} \geq\left|x_{1}\right|^{\frac{2}{2-\alpha}}
$$

Also, the following can be obtained from (33):

$$
\frac{M_{\tilde{R}}}{2} \max \left\{1, \mu_{2}(2-\alpha)\right\}\left(\left|x_{1}\right|^{\frac{2}{2-\alpha}}+x_{2}^{2}\right) \geq V_{\tilde{R}}\left(x_{1}, x_{2}\right)
$$

Hence, the following inequality is then obtained for $\left|x_{1}\right| \geq 1$ by combining (44), (46) and (47):

$$
\dot{V}_{\tilde{R}} \leq-\bar{\kappa}_{1} V_{\tilde{R}}
$$

where,

$$
\bar{\kappa}_{1}=\frac{2 c_{\tilde{R}}}{M_{\tilde{R}} \max \left\{1, \mu_{2}(2-\alpha)\right\}}>0 .
$$

Case 2: $\left|x_{1}\right|<1$ :

Noting that the following inequalities hold true for $\left|x_{1}\right|<1$,

$$
\left|x_{1}\right|^{\frac{10-4 \alpha}{2-\alpha}}>\left|x_{1}\right|^{\frac{2 \gamma}{2-\alpha}} \Leftrightarrow \frac{10-4 \alpha}{2-\alpha}<\frac{2 \gamma}{2-\alpha} \Leftrightarrow \gamma>5-2 \alpha,
$$

and for some $\gamma>5-2 \alpha$. Noting that $5-2 \alpha<\frac{11}{3}$ always holds true due to $\alpha \in\left(\frac{2}{3}, 1\right), \gamma \geq \frac{11}{3}$ is a valid choice. In the following, $\gamma=4$ is chosen. It can be seen that the following equality holds true:

$$
\begin{aligned}
\left(\left|x_{1}\right|^{\frac{2}{2-\alpha}}+x_{2}^{2}\right)^{4}= & \left|x_{1}\right|^{\frac{8}{2-\alpha}}+4\left|x_{1}\right|^{\frac{6}{2-\alpha}} x_{2}^{2}+6\left|x_{1}\right|^{\frac{4}{2-\alpha}} x_{2}^{4} \\
& +4\left|x_{1}\right|^{\frac{2}{2-\alpha}} x_{2}^{6}+x_{2}^{8} \\
\leq & \max \left\{\rho^{2 \alpha-1}, K_{2}\right\}\left(\left|x_{1}\right|^{\frac{10-4 \alpha}{2-\alpha}}+x_{2}^{2}\right)^{(51)}
\end{aligned}
$$

where the bounds (27) has been utilised resulting in the following definition of $K_{2}$ :

$$
K_{2}=\max \left\{4 \rho^{3}, 6 \rho^{2}(2 \tilde{R}), 4 \rho(2 \tilde{R})^{2},(2 \tilde{R})^{3}\right\}>0
$$

Note that the following can be obtained from (33):

$$
\left(\frac{M_{\tilde{R}}}{2} \max \left\{1, \mu_{2}(2-\alpha)\right\}\left(\left|x_{1}\right|^{\frac{2}{2-\alpha}}+x_{2}^{2}\right)\right)^{4} \geq\left(V_{\tilde{R}}\left(x_{1}, x_{2}\right)\right)^{4}
$$

Then, the following can be obtained by combining (44), (51) and (53):

$$
\dot{V}_{\tilde{R}}\left(x_{1}, x_{2}\right) \leq-c_{\tilde{R}}\left(\left|x_{1}\right|^{\frac{10-4 \alpha}{2-\alpha}}+x_{2}^{2}\right) \leq-\bar{\kappa}_{2}\left(V_{\tilde{R}}\right)^{4}
$$

where,

$$
\bar{\kappa}_{2}=\frac{c_{\tilde{R}}}{\left(\frac{M_{\tilde{R}}}{2} \max \left\{1, \mu_{2}(2-\alpha)\right\}\right)^{4} \max \left\{\rho^{2 \alpha-1}, K_{2}\right\}}>0 .
$$

Hence, the desired uniform negative definiteness (21) is obtained by combining (48) and (54) as follows:

$$
W_{\tilde{R}}\left(x_{1}, x_{2}\right)=\min \left\{\bar{\kappa}_{1} V_{\tilde{R}}, \bar{\kappa}_{2}\left(V_{\tilde{R}}\right)^{4}\right\}
$$

3. Global Equiuniform Asymptotic Stability Since the inequality (21) holds on the solutions of the uncertain system (11), (12), initialized within the compact set (20), the decay of the function $V_{\tilde{R}}\left(x_{1}, x_{2}\right)$ can be found by considering the majorant solution $v(t)$ of $V_{\tilde{R}}$ as follows:

$$
\dot{v}(t)= \begin{cases}-\bar{\kappa}_{1} v(t), & \text { if }\left|x_{1}\right| \geq 1 ; \\ -\bar{\kappa}_{2} v^{\gamma}, & \text { if }\left|x_{1}\right|<1 .\end{cases}
$$

where, $\gamma>5-2 \alpha$ is introduced for generality. A more conservative decay than that in (57) can be computed. There are two possible sub-cases, namely, $v(t) \geq 1$ and $v(t)<1$ for each of the cases $\left|x_{1}\right| \geq 1$ and $\left|x_{2}\right|<1$. The following expressions hold true for a positive definite function $v(t)$ and a scalar $\gamma>1$ :

$$
\begin{array}{ll}
v(t)^{\gamma} \geq v(t) \Rightarrow-v(t)^{\gamma} \leq-v(t) & \text { if } v(t) \geq 1 \\
v(t)^{\gamma} \leq v(t) \Rightarrow-v(t) \leq-v(t)^{\gamma} & \text { if } v(t)<1 .
\end{array}
$$

Hence, the decay (57) is modified by utilising (58) independent of the magnitude of $\left|x_{1}\right|$ and dependent on $v(t)$ as follows :

$$
\dot{v}(t)= \begin{cases}-\bar{\kappa} v, & \text { if } v(t) \geq 1 ; \\ -\bar{\kappa} v^{\gamma}, & \text { if } v(t)<1 .\end{cases}
$$


where

$$
\bar{\kappa}=\min \left\{\bar{\kappa}_{1}, \bar{\kappa}_{2}\right\} \quad>0 .
$$

The solution for the case $v(t)<1$ can be obtained as follows:

$$
\int_{v_{0}}^{v(t)} \frac{\mathrm{d} \zeta(t)}{\zeta \gamma}=-\bar{\kappa} \int_{t_{1}}^{t} \mathrm{~d} \tau
$$

where $v_{0}=v\left(t_{1}\right)$ where $t_{1}$ is the time instant when the solution $v(t)$ satisfies the condition $v(t)=1$. The general solution of $v(t)$ of (59) can then be obtained as follows:

$$
v(t)= \begin{cases}v\left(t_{0}\right) e^{-\bar{\kappa}\left(t-t_{0}\right)}, & \text { if } v(t) \geq 1 \\ v\left(t_{1}\right)\left(\frac{1}{\bar{\kappa}\left(t-t_{1}\right)(\gamma-1) v_{0}^{\gamma-1}+1}\right)^{\frac{1}{\gamma-1}}, & \text { if } v(t)<1\end{cases}
$$

It is noted that $t_{1}=t_{0}$ if $v\left(t_{0}\right) \leq 1$. It can be easily seen that the solution $v(t) \rightarrow 0$ as $t \rightarrow \infty$ and that the decay rate depends on the gain parameters $\mu_{1}, \mu_{2}$ and bound $M$ on the disturbance $\omega(x, t)$. On the compact set (20), the following inequality holds (see (31), (33)):

$$
L_{\tilde{R}} V\left(x_{1}, x_{2}\right) \leq V_{\tilde{R}}\left(x_{1}, x_{2}\right) \leq M_{\tilde{R}} V\left(x_{1}, x_{2}\right)
$$

for all $\left(x_{1}, x_{2}\right) \in D_{\tilde{R}}$ and positive constants $L_{\tilde{R}}, M_{\tilde{R}}$. The above inequalities (62) and (63) ensure that the globally radially unbounded function $V\left(x_{1}, x_{2}\right)$ decays exponentially

$$
\begin{aligned}
& V\left(x_{1}(t), x_{2}(t)\right) \\
& \leq \begin{cases}L_{\tilde{R}}^{-1} M_{\tilde{R}} \tilde{R} e^{-\bar{\kappa}\left(t-t_{0}\right)}, & \text { if } V_{\tilde{R}} \geq 1 ; \\
L_{\tilde{R}}^{-1} M_{\tilde{R}} \tilde{R}\left(\frac{1}{\bar{\kappa}\left(t-t_{1}\right)(\gamma-1) v_{0}^{\gamma-1}+1}\right)^{\frac{1}{\gamma-1}}, & \text { if } V_{\tilde{R}}<1 .\end{cases}
\end{aligned}
$$

on the solutions of (11), (12) uniformly in $\omega$ and the initial data, located within an arbitrarily large set (20). This proves that the uncertain system (11), (12) is globally equiuniformly asymptotically stable around the origin $\left(x_{1}, x_{2}\right)=(0,0)$. 4. Global Equiuniform Finite Time Stability.

The uncertainty $\omega\left(x_{1}, x_{2}, t\right)$ in the right hand side of the system (11), (12) is uniformly bounded by $M\left|x_{2}\right|^{\alpha}$. The feedback is globally homogeneous with homogeneity degree $q=-1$ with respect to dilation $\left(r_{1}, r_{2}\right)=\left(\frac{2-\alpha}{1-\alpha}, \frac{1}{1-\alpha}\right)$. In the presence of continuous disturbances $\omega\left(x_{1}, x_{2}, t\right)$, Lemma 1 proves that the closed-loop system (11), (12) is homogeneous of degree $q=-1$ with respect to dilations $\left(r_{1}, r_{2}\right)=\left(\frac{2-\alpha}{1-\alpha}, \frac{1}{1-\alpha}\right)$. Thus, coupling the homogeneity of the perturbed system (11), (12) within the arbitrarily large compact set (20), with the global equiuniform asymptotic stability of the system (11), (12), it is obtained that the closed-loop system (11), (12) is globally equiuniformly finite time stable according to [10, Theorem $3.1]$.

\section{CONCLUSION}

The paper studied robustness of existing finite time continuous homogeneous controllers to time varying disturbances. A detailed proof of the uniform finite time stability of the perturbed double integrator was established by identifying a class of $C^{1}$ smooth semi-global Lyapunov functions. A possible future direction is to study equiuniform finite time stability in the general dimension $n$ in the presence of timevarying disturbances.

\section{REFERENCES}

[1] V. Haimo, "Finite time controllers," SIAM Journal on Control and Optimization, vol. 24, no. 4, pp. 760-770, 1986.

[2] S. Bhat and D. Bernstein, "Finite-time stability of continuous autonomous systems," SIAM J. Control Optim., vol. 38, no. 3, pp. 751$766,2000$.

[3] _ , "Finite-time stability of homogeneous systems," in Proceedings of the American Control Conference, 1997.

[4] — , "Continuous finite-time stabilization of the translational and rotational double integrators," Automatic Control, IEEE Transactions on, vol. 43, no. 5, pp. 678-682, May 1998.

[5] Y. Hong, J. Huang, and Y. Xu, "On an output feedback finitetime stabilization problem," IEEE Transactions on Automatic Control, vol. 46, no. 2, pp. $305-309,2001$.

[6] E. Moulay and W. Perruquetti, "Finite time stability and stabilization of a class of continuous systems," Journal of Mathematical Analysis and Applications, vol. 323, no. 2, pp. $1430-1443,2006$.

[7] L. Rosier, "Homogeneous lyapunov function for homogeneous continuous vector field," Systems \& Control Letters, vol. 19, no. 6, pp. $467-473,1992$.

[8] M. Kawski, "Stability and nilpotent approximations," in Proceedings of the 27th IEEE Conference on Decision and Control, Dec 1988, pp. 1244-1248.

[9] S. Bhat and D. Bernstein, "Geometric homogeneity with applications to finite-time stability," Mathematics of Control, Signals, and Systems (MCSS), vol. 17, pp. 101-127, 2005.

[10] Y. Orlov, "Finite time stability and robust control synthesis of uncertain switched systems," SIAM Journal on Control and Optimization, vol. 43, no. 4, pp. 1253-1271, 2005.

[11] W. Haddad, S. Nersesov, and L. Du, "Finite-time stability for timevarying nonlinear dynamical systems," in American Control Conference, june 2008, pp. $4135-4139$.

[12] C. Qian, "A homogeneous domination approach for global output feedback stabilization of a class of nonlinear systems," in American Control Conference, vol. 7, june 2005, pp. $4708-4715$.

[13] V. Andrieu, L. Praly, and A. Astolfi, "Homogenous approximation, recursive observer design, and output feedback," SIAM Journal on Control and Optimization, vol. 47, no. 4, pp. 1814-1850, 2008.

[14] X. Zhang, G. Feng, and Y. Sun, "Finite-time stabilization by state feedback control for a class of time-varying nonlinear systems," Automatica, vol. 48, no. 3, pp. 499 - 504, 2012.

[15] E. Bernuau, W. Perruquetti, D. Efimov, and E. Moulay, "Finite-time output stabilization of the double integrator," in $51^{\text {st }}$ IEEE Annual Conference on Decision and Control (CDC), Dec 2012, pp. 59065911.

[16] R. Santiesteban, "Time convergence estimation of a perturbed double integrator: Family of continuous sliding mode based output feedback synthesis," in European Control Conference (ECC), July 2013, pp. 3764-3769.

[17] A. Chalanga, S. Kamal, and B. Bandyopadhyay, "Continuous integral sliding mode control: A chattering free approach," in IEEE International Symposium on Industrial Electronics (ISIE), 2013, pp. 1-6.

[18] H. B. Oza, Y. V. Orlov, and S. K. Spurgeon, "Continuous uniform finite time stabilisation of planar controllable systems," SIAM Journal of Control and Optimization, Submitted in May 2012.

[19] A.F.Filippov, Differential Equations with Discontinuous Righ thand Sides, ser. Mathematics and its Applications. Springer, 1988, vol. 18.

[20] M. Kawski, "Families of dilations and asymptotic stability," in Analysis of controlled dynamical systems (Lyon, 1990), ser. Progr. Systems Control Theory. Boston, MA: Birkhäuser Boston, 1991, vol. 8, pp. 285-294.

[21] Y. Orlov, Y. Aoustin, and C. Chevallereau, "Finite time stabilization of a perturbed double integrator - part I: Continuous sliding mode-based output feedback synthesis," IEEE Transactions on Automatic Control, vol. 56, no. 3, 2010.

[22] J. Alvarez, Y. Orlov, and L. Acho, "An invariance principle for discontinuous dynamic systems with applications to a coulumb friction oscillator," Journal of Dynamic Systems, Measurement and Control, vol. 74, pp. $190-198,2000$.

[23] D. Shevitz and B. Paden, "Lyapunov stability theory of nonsmooth systems," Automatic Control, IEEE Transactions on, vol. 39, no. 9, pp. 1910 -1914, sep 1994. 